\title{
A Systemic Review of the Materials Used in Primary Teeth Pulpotomy in Children
}

\author{
Rajendran Ganesh ${ }^{1 *}$, Selvakumar $\mathbf{H}^{2}$ and R Kolappan ${ }^{3}$ \\ ${ }^{1}$ Assistant Professor, Paediatric Dentist, Department of Preventive Dental Science, Al Baha University, Al Baha, Saudi Arabia \\ ${ }_{2}^{2}$ Associate Professor, Department of Paediatric Dentistry, Sri Ramachandra Dental College and Hospital, Chennai, India \\ ${ }^{3}$ Oral Medicine and Maxillofacial Radiologist, Senior Lecturer, Dept of Oral Medicine, Kannur Dental College, India \\ *Corresponding Author: Rajendran Ganesh, Assistant Professor, Paediatric Dentist, Department of Preventive Dental Science, Al Baha \\ University, Al Baha, Saudi Arabia.
}

Received: October 14, 2019; Published: November 05, 2019

DOI: $10.31080 /$ ASDS.2019.03.0692

\begin{abstract}
Traditionally, the term pulpotomy has implied removal of pulp tissue to the cervical line. However, the depth to which tissue is removed is determined by clinical judgement. A primary goal of all restorative treatment is to maintain pulp vitality so that normal root development or paedogenesis can occur. If pulpal exposure occurs, then a pulpotomy procedure aims to preserve pulp vitality to allow for normal root development. Historically, calcium hydroxide has been the material of choice for pulpotomy procedures. Recently, an alternative material called mineral trioxide aggregate (MTA) has demonstrated the ability to induce hard-tissue formation in pulpal tissue. This review describes the different materials used in pulpotomy and their advantages, disadvantages and its success in pulpotomy.
\end{abstract}

Keywords: Pulpotomy, Primary Teeth

\section{Introduction}

The pulpotomy procedure involves removing pulp tissue that has inflammatory or degenerative changes leaving intact the remaining vital tissue, which is then covered with a pulp capping agent to promote healing at the amputation site.

In 1885 when Leptowskis introduced formalin as a mummifying agent, better fixation of pulp stumps was assured. Since 1886, gold foil was advocated as a protective covering over an exposed pulp and Bodecker for making his biological studies of many of the pulp capping. In 1898 Gysi of the dental institute of Zurich developed as less irritating preparations containing paraformaldehyde as the principle ingredient. In 1904, Buckley introduced formicaries or tricerion formalin, which is commonly, used material for pulpotomy today. In 1930 it was shown by Herman that a living pulp amputated and covered with calcium mixture called calyl would repair itself by riding across the cut pulp with reparative dentin [1]. Rolling and Hansen (1978) showed that form cresol causes chronic inflammation and necrotic tissues in contrast to 70 - 90 \% success rate. Reumpling., et al. (1983) have demonstrated the use of electrosurgery for pulpotomy [2].

\section{Classification}

Pulpotomy can be classified according to the treatment objectives (Don Ranley 1994 [3]).

1. Devitalization pulpotomy (mummification)

a) Form cresol pulpotomy.

b) Electrosurgical pulpotomy.

c) Laser pulpotomy.

2. Preservation (minimal devitalization, non inductive)

a) Glutaraldehyde.

b) Ferric sulphate.

3. Regeneration (Inductive, Reparative)

a) Calcium hydroxide.

b) MTA. 
The first pulpotomy technique was advocated by Sweet in 1930 as a multi- visit technique for vital as well as for non - vital teeth. Then Kennedy D.B 1986 reduced the multi visit approach to 2 visits in vital teeth [4].

\section{Formocresol pulpotomy}

Formocresol was introduced in 1904 by Buckley, who contended that equal parts of formalin and tricerion would react chemically with the intermediate and end products of pulpal inflammation to form a new colourless and non infective compound of a harmless nature [2].

The use of formocresol as a pulpal medicament was first introduced by Sweet in 1904. By 1955, Sweet claimed 97\% dentinal success in 16, 651 cases [5]. Berger (1965) conducted a study on 30 teeth with full strength formocresol ( $5 \mathrm{~min}$ ) and showed a clinical success rate of $100 \%$ and a radiographic success rate of $97 \%$. Beaver (1966) showed a radiographic success of $93 \%$ and clinical success rate of $100 \%$ (full strength formoacresol-5min). Redig (1968) conducted a study with 5 minute full strength formocresol application on 20 teeth and showed a radiographic and a clinical success rate of $85 \%$, and a 2 appointment full strength formocresol pulpotomy in 20 teeth showed a clinical and a radiographic success rate of $90 \%$ [6]. Rolling (1975) carried out pulpotomy in 98 teeth with full strength formocresol (5 min); found 77\% clinical success rate after 36 months and radiographic success of $91 \%$ after 3 months, $83 \%$ after 12 months, 78\% after 24 months and 70\% after 36 months [6]. Fuks [7] conducted a 1:5 dilution formocresol pulpotomy ( $5 \mathrm{~min}$ ) in 70 teeth which showed a clinical success rate of $100 \%$ and a radiographic success of $94 \%$.

\section{Glutaraldehyde}

It is a bifunctional reagent which allows it to form strong intra and intermolecular protein bonds leading to superior fixation by cross linkages. Glutaraldehyde has been suggested as an alternative to form cresol in primary tooth pulpotomy. Histologic assessment of glutaraldehyde pulpotomy technique by Kopel., et al. revealed that a $2 \%$ solution results in maintenance of pulp vitality beneath an initial zone of fixation. Clinical results on human primary teeth treated by $2 \%$ glutaraldehyde pulpotomy demonstrated $96 \%$ success over the first 2 years [8].

Ferric sulphate

Ferric sulphate $(15.5 \%)$ causes coagulation of the tissues at the entrances of the root canals, in much the same way as formocresol.
Success has been claimed with this technique using combined radiographic and clinical assessment after a 1-year follow-up. Fuks., et al. [9] found similar results between form cresol and ferric sulphate in inflammation response, periradicular or interradicular abscesses, root resorption, and dentin bridge formation. Fei., et al. [10] (1991) found ferric sulphate to produce greater clinical and radiographical success after 1 year than did form cresol. More recently Smith., et al. (2000) evaluated the long term success rates of ferric sulphate pulpotomies in dental practice [6].

Lasers

Typically, the carbon dioxide laser, have been used experimentally for pulpotomy in dogs (Shoji., et al.). It was claimed that the laser caused no pulpal damage but obviously more research is needed, to gather with clinical trials to determine the effectiveness and the type of laser best suited to pulpotomy technique [6].

\section{Cautery (Electrosurgery)}

It has been suggested by Ruempling., et al. 1983 to fix radicular pulp tissue after amputation of the coronal pulp. Pulpal response was as favourable as that after form cresol. These However, further work by Schulman., et al. (1987) noted pathological resorption and periapical / furcal pathology. As with lasers, further work on the use of cautery is needed [6].

\section{Calcium hydroxide}

Calcium hydroxide is a white, crystalline, slightly soluble basic salt that dissociates into calcium ions and hydroxyl ions in solution and exhibits a high alkalinity ( $\mathrm{pH} 11)$. Codman was the first to use calcium hydroxide in pulpal treatment [11]. Dentists also use calcium hydroxide because of its antimicrobial properties [12] and its ability to induce hard-tissue formation. Researchers have shown that calcium hydroxide forms a dentin bridge when placed in contact with pulpal tissues [13]. The antimicrobial effect of pulpotomy materials relates to the ability of the material to kill existing bacteria and prevent the future leakage of bacteria from the oral environment into the pulp [14]. The antimicrobial properties of calcium hydroxide are derived from several factors [15].

The high pH produces an environment that is not conducive to bacteria growth. There are three mechanisms by which calcium hydroxide induces bacterial lysis: the hydroxyl ions destroy phospholipids so the cellular membrane is destroyed; the high alkalinity breaks down ionic bonds so that bacterial proteins are denatured; the hydroxyl ions react with bacterial DNA, inhibiting 
replication [16]. Several studies have assessed the outcomes of calcium hydroxide in vital pulpal treatment. Generally, as the length of the follow-up period increased, the success rate decreased. At the five- and 10-year follow-ups, pulp capping of curiously exposed teeth resulted in a failure rate of 44.5 percent and 79.7 percent, respectively. In teeth with carious exposures treated with calcium hydroxide pulpotomy, healing teeth ranged from approximately 50 to 92 percent. A study examining 41 teeth with carious pulpal exposures (with or without peri radicular changes seen on radiographic examination) treated with pulpotomy and calcium hydroxide reported a healing rate of 87 to 79 percent. The healing rate in teeth with pre-existing pain, however, was approximately 50 percent [17]. The follow-up period varied from six to eight months. In another study, 37 posterior teeth with deep carious lesions that were exposed during caries removal were treated with calcium hydroxide pulpotomy. The patients ranged in age from 6 to 15 years. Six teeth had widened periodontal ligament spaces periradicularly and a history of temporary pain. None of the other teeth had signs and symptoms of pulpal or peri radicular inflammation or infection. The healing rate was 89 percent, and the follow-up period ranged from 24 to 140 months, with a mean of 56 months. The healing rates in teeth with traumatic exposures that were treated with calcium hydroxide ranged from 72 to 96 percent [18].

MTA

MTA is composed of tricalcium silicate, tricalcium aluminate, tricalcium oxide and silicate oxide. It is available commercially as ProRoot MTA (Dentsply Tulsa Dental, Tulsa, Okla.) and has been advocated for use in vital pulp therapy [19]. It has a compressive strength equal to Intermediate Restorative Material but less than that of amalgam. MTA has demonstrated the ability to induce hard-tissue formation in pulpal tissues, and it promotes rapid cell growth in vitro. Compared with calcium hydroxide.

MTA has demonstrated a greater ability to maintain the integrity of pulp tissue. Histologic evaluation of pulpal tissue in animals and humans demonstrated that MTA produces a thicker dentinal bridge, less inflammation, less hyperaemia and less pulpal necrosis compared with calcium hydroxide. MTA also appears to induce the formation of a dentin bridge at a faster rate than does calcium hydroxide [20]. The process by which MTA acts to induce dentin bridge formation, however, is not known. Holland and colleagues theorized that the tricalcium oxide in MTA reacts with tissue fluids to form calcium hydroxide, resulting in hard-tissue formation. The ability of MTA to resist the penetration of microorganisms appears to be high [18]. In leakage studies, MTA frequently performs better than amalgam, IRM Furthermore; the presence of blood has little impact on the degree of leakage [21].

To date, the clinical assessment of MTA has been restricted to case reports. In one, researchers performed partial pulpotomy in two cases of dens evaginates. After six months, researchers removed the teeth as part of planned orthodontic treatment. Histologic examination of these teeth showed an apparent continuous dentin bridge formation in both teeth, and the pulps were free of inflammation [22]. Resin-modified glass ionomers have improved wear resistance compared to the original glass ionomers and are appropriate restorative materials after pulpotomy of primary teeth [22]. Another systemic review reported that no statistical significant difference in the clinical success rates of pulpotomies and pulpectomies in the pulp treatment of carious vital pulp exposure in primary incisors while radiographically, pulpectomy may have a higher risk for radiographic failure than pulpotomy [22]. Hence priority teaching of pulpotomy as a treatment option for vital pulp exposure in primary incisors in paediatric dentistry residency programs should be done.

\section{Conclusion}

The rationale for primary teeth pulpotomy has developed out of extensive clinical studies and improved histological techniques. Ongoing research will result in modification that will enhance treatment outcomes. A successful pulpotomy outcome should be based on freedom from pathologic root resorption; maintenance of the primary teeth in an infection free state to hold space for the eruption of its permanent successor. The material, MTA may be useful as a substitute for other materials in pulpotomy procedures.

\section{Bibliography}

1. Ranly DM. "Pulpotomy therapy in primary teeth new modalities for old rationales". Quintessence International 16.6 (1994): 403-409.

2. Camp JH. "Pulp therapy for primary and young permanent teeth". Dental Clinics of North America 28 (1984): 651-668.

3. Dm Ranley and Garcia- Godoy F. "Current and potential pulp therapies for primary and young permanent teeth". Journal of Dentistry 28 (2000): 153-161. 
4. M E J Curzon., et al. "Kennedy's Pediatric Operative Dentistry". 4th edition 143.

5. Frigoletto RT. "Pulp therapy in Pedodontics". Journal of the American Dental Association 86 (1973): 1344.

6. Srinivasan V., et al. "Is there life after Bucley's formocresol? Part I- a narrative of alternate interventions and materials". International Journal of Paediatric Dentistry 16.2 (2006): 117 127.

7. Anna B Fuks., et al. "Root fillings with Endoflas in primary teeth: a retrospective study". Journal of Clinical Pediatric Dentistry 27.1 (2002): 41-45.

8. FEI., et al. "Clinical study of ferric sulfate as a pulpotomy agent in primary teeth". Pediatric Dentistry 13 (1995): 327-332.

9. Codman WW. "Ossification of the pulp of a tooth". Newsletter 6 (1981): 90.

10. Safavi KE and Nichols FC. "Effect of calcium hydroxide on bacterial lipopolysaccharide”. Journal of Endodontics 19.2 (1993): 76-78.

11. Tronstad L. "Reaction of the exposed pulp to Dycal treatment". Oral Surgery, Oral Medicine, Oral Pathology 38 (1974): 945953.

12. Pitt Ford TR. "Pulpal response to a calcium hydroxide material capping exposures". Oral Surgery, Oral Medicine, Oral Pathology 59.2 (1985): 194-197.

13. Tagger M and Tagger E. "Pulp capping in monkeys with Reolit and Life, two calcium hydroxide bases with different $\mathrm{pH}^{\mathrm{N}}$. Journal of Endodontics 11 (1985): 394-400.

14. Siqueira JF Jr and Lopes HP. "Mechanisms of antimicrobial activity calcium hydroxide: a critical review". International Endodontic Journal 32 (1999): 361-369.

15. Mejare I and Cvek M. "Partial pulpotomy in young permanent teeth with deep carious lesions". Endodontics and Dental Traumatology 9 (1993): 238-242.

16. Cvek M. "A clinical report on partial pulpotomy and capping with calcium hydroxide in permanent incisors with complicated crown fracture". Journal of Endodontics 4 (1978): 232237.
17. Ford TR., et al. "Using mineral trioxide aggregate as a pulpcapping material”. JADA 127.10 (1996): 1491-1494.

18. Torabinejad M and Chivian N. "Clinical applications of mineral trioxide aggregate”. Journal of Endodontics 25.3 (1999): 197205.

19. Torabinejad M., et al. "Bacterial leakage of mineral trioxide aggregate as a root-end filling material". Journal of Endodontics 21 (1995): 109-112.

20. Torabinejad M., et al. "Dye leakage of four root end filling materials: effects of blood contamination". Journal of Endodontics 20 (1994): 159-163.

21. Mohammad N., et al. "S1Comparative Evaluation of the Fracture Strength of Pulpotomized Primary Molars: An In Vitro Study". International Journal of Clinical Pediatric Dentistry 12.1 (2019): 5-9.

22. Gadallah Lamia., et al. "Pulpotomy versus pulpectomy in the treatment of vital pulp exposure in primary incisors. A systematic review and meta-analysis". F1000 Research 7 (2018): 1560.

\section{Volume 3 Issue 12 December 2019}

(C) All rights are reserved by Rajendran Ganesh., et al. 